This item was submitted to Loughborough's Research Repository by the author.

Items in Figshare are protected by copyright, with all rights reserved, unless otherwise indicated.

\title{
Possible high-pressure orbital quantum criticality and an emergent resistive phase in $\mathrm{PbRuO3}$
}

PLEASE CITE THE PUBLISHED VERSION

http://dx.doi.org/10.1103/PhysRevB.87.165130

\section{PUBLISHER}

(C) American Physical Society

\section{VERSION}

VoR (Version of Record)

\section{PUBLISHER STATEMENT}

This work is made available according to the conditions of the Creative Commons Attribution-NonCommercialNoDerivatives 4.0 International (CC BY-NC-ND 4.0) licence. Full details of this licence are available at: https://creativecommons.org/licenses/by-nc-nd/4.0/

\section{LICENCE}

CC BY-NC-ND 4.0

\section{REPOSITORY RECORD}

Kusmartseva, Anna F., Alexandra Sinclair, Jennifer A. Rodgers, Simon A.J. Kimber, and J. Paul Attfield. 2019. "Possible High-pressure Orbital Quantum Criticality and an Emergent Resistive Phase in Pbruo3". figshare. https://hdl.handle.net/2134/17360. 


\title{
Possible high-pressure orbital quantum criticality and an emergent resistive phase in $\mathrm{PbRuO}_{3}$
}

\author{
Anna F. Kusmartseva, ${ }^{1,2}$ Alexandra Sinclair, ${ }^{1}$ Jennifer A. Rodgers, ${ }^{1}$ Simon A. J. Kimber, ${ }^{3}$ and J. Paul Attfield ${ }^{1, *}$ \\ ${ }^{1}$ Centre for Science at Extreme Conditions and School of Chemistry, University of Edinburgh, King's Buildings, Mayfield Road, \\ Edinburgh EH9 3JZ, United Kingdom \\ ${ }^{2}$ Department of Physics, Loughborough University, Leicestershire LE11 3TU, United Kingdom \\ ${ }^{3}$ European Synchrotron Radiation Facility, Boite Postale 220, 38043 Grenoble Cedex, France \\ (Received 30 November 2012; revised manuscript received 21 February 2013; published 23 April 2013)
}

\begin{abstract}
The orbital ordering transition in the metallic perovskite $\mathrm{PbRuO}_{3}$ is suppressed from $90 \mathrm{~K}$ at ambient pressure towards zero temperature at $50 \mathrm{kbar}$, where non-Fermi liquid resistivity with a temperature exponent $n=1.6$ is observed. This evidences a possible quantum critical point brought about by orbital fluctuations, rather than spin fluctuations as observed in $\mathrm{Sr}_{3} \mathrm{Ru}_{2} \mathrm{O}_{7}$ and heavy fermion conductors. An anomalous increase of resistivity is observed at pressures above $\sim 100 \mathrm{kbar}$, and a transition to a more resistive, possibly semiconducting, phase is observed at $300 \mathrm{kbar}$ and ambient temperature.
\end{abstract}

DOI: 10.1103/PhysRevB.87.165130

PACS number(s): 75.25.Dk, 72.15.-v, 72.80.Ga

\section{INTRODUCTION}

Quantum critical phenomena have been reported in many correlated electron materials over the last decade. ${ }^{1,2}$ Tuning a phase transition towards zero temperature leads to a quantum critical point (QCP) around which quantum mechanical fluctuations dominate over thermal effects and alternative ground states such as unconventional superconducting phases are observed. New orders can emerge as the system is further tuned beyond the QCP and may persist to ambient temperature. QCPs are usually associated with suppression of a second-order transition, but are also reported at suppressed first-order transitions in some strongly correlated materials such as the heavy fermion ferromagnets $\mathrm{UGe}_{2}$ (Ref. 3 ) and URhGe. ${ }^{4}$ Transition metal oxides show many exotic conducting states and phase transitions ${ }^{5}$ so a variety of quantum critical phenomena may be expected. QCPs in conducting oxides have been accessed by suppressing magnetic transitions, and are implicated in the emergence of superconductivity in doped antiferromagnetic cuprates, but the best established example is in the bilayer ruthenate $\mathrm{Sr}_{3} \mathrm{Ru}_{2} \mathrm{O}_{7}{ }^{6,7}$ This has a magnetic field-induced QCP at $8 \mathrm{~T}$ resulting from suppression of a metamagnetic transition. A subtle lattice distortion attributed to nematic orbital ordering correlations has recently been discovered in the 0.2 -T-wide phase that emerges around the QCP. ${ }^{8}$

Localized $\mathrm{Ru}^{4+}$ states have spin $S=1$ and a triple orbital degeneracy arising from the $t_{2 \mathrm{~g}}{ }^{4} d$-electron configuration. Many ruthenate perovskites are metallic with Fermi surfaces resulting from hybridization between oxygen $2 p$ and ruthenium $4 d$ levels, with spin or orbital instabilities leading to diverse and competing ground states. The $\mathrm{Ru}-\mathrm{O}-$ $\mathrm{Ru}$ bond angle has been identified as an important control parameter that may be tuned using cation substitutions or pressure $;{ }^{9}$ for example, layered $\mathrm{Sr}_{2} \mathrm{RuO}_{4}$ is an unconventional, $p$-wave superconductor ${ }^{10}$ at low temperatures, but $\mathrm{Ca}_{2} \mathrm{RuO}_{4}$ is an antiferromagnetic insulator. ${ }^{11}$ There is some evidence for suppression of antiferromagnetism and emergence of superconductivity associated with a QCP in $\mathrm{Ca}_{2} \mathrm{RuO}_{4}$ around $\sim 100$ kbar pressure. ${ }^{12,13}$ Among the cubic-type ruthenate perovskites, $\mathrm{SrRuO}_{3}$ and $\mathrm{BaRuO}_{3}$ are itinerant ferromagnets ${ }^{14-16}$ but $\mathrm{CaRuO}_{3}$ remains a paramagnetic metal with a large mass enhancement at low temperature. ${ }^{17,18} \mathrm{PbRuO}_{3}$ is a paramag- netic metal and displays orbital ordering transition at $90 \mathrm{~K}$, where the superstructure space group symmetry shows an unconventional increase from Pnma to Imma on cooling. ${ }^{19,20}$ No spin ordering transition is observed down to $1.5 \mathrm{~K}$. A study of the $\mathrm{Sr}_{1-x} \mathrm{~Pb}_{x} \mathrm{RuO}_{3}$ system reported two possible QCPs, at $x=0.6$ and 0.9 , based on resistivity measurements of ceramic samples. $^{21}$ Here we report evidence for a possible QCP in $\mathrm{PbRuO}_{3}$, induced by pressure suppression of orbital order, and the emergence of resistive correlations and a structural phase at higher pressures.

\section{EXPERIMENTAL RESULTS}

The perovskite $\mathrm{PbRuO}_{3}$ requires high pressures for synthesis. $^{22}$ Samples were synthesized at $11 \mathrm{GPa}$ and $1100{ }^{\circ} \mathrm{C}$ using a Walker-type multianvil press, as described previously. ${ }^{19}$ A high-pressure $\mathrm{X}$-ray diffraction study was carried out on instrument ID09A at the European Synchrotron Radiation Facility using a wavelength of $0.4144360 \AA$. Polycrystalline $\mathrm{PbRuO}_{3}$ was contained in a diamond anvil cell (DAC) using helium as a hydrostatic pressure transmitting medium and a ruby as a pressure calibrant. Diffraction profiles were fitted using the GSAS program. ${ }^{23}$ However, quantitative intensities for structure refinement were not obtained due to sample granularity or pressure-induced texturing, so only lattice parameters were extracted from the data.

X-ray diffraction profiles at pressures up to $125 \mathrm{kbar}$ and temperatures of $20-200 \mathrm{~K}$ in a He-pumped cryostat were used to explore the suppression of the transition from the high-temperature Pnma superstructure (phase I) to the low-temperature, orbitally ordered Imma superstructure (phase II). The I-II transition is observed at $0,10,15$, and $30 \mathrm{kbar}$ from discontinuities in lattice parameter plots (Fig. 1), and so remains first order up to at least $30 \mathrm{kbar}$, and is suppressed to below $20 \mathrm{~K}$ between 30 and 50 kbar. Suppression of the transition to zero temperature is predicted to be at $\sim 45$ $55 \mathrm{kbar}$ - linear extrapolation from the 0-30 kbar transition temperatures gives a value of $55 \mathrm{kbar}$ as shown on the phase diagram in Fig. 2.

Small $\mathrm{PbRuO}_{3}$ single crystals of approximately plateletlike geometry, with longest dimension $\sim 100-150 \mu \mathrm{m}$ and 


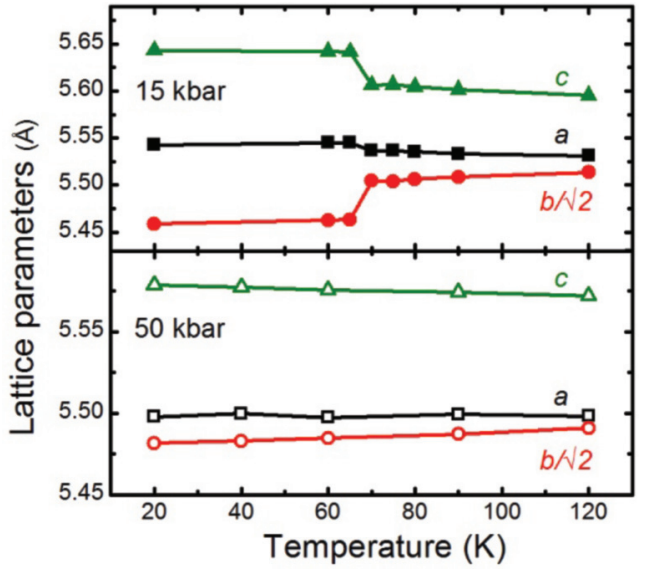

FIG. 1. (Color online) Low-temperature variation of the orthorhombic lattice parameters for $\mathrm{PbRuO}_{3}$ at representative pressures. The discontinuity observed at $65 \mathrm{~K}$ in the $15 \mathrm{kbar}$ data evidences the Imma to Pnma orbital order transition which is suppressed near 50 kbar.

thickness $\sim 50-80 \mu \mathrm{m}$, were isolated from some synthesis runs, and their resistivities were measured over temperatures $1.8-300 \mathrm{~K}$ at high pressures in several DAC experiments. A four-terminal arrangement of gold leads was used, with a two-part Stycast epoxy mixed with $\mathrm{Al}_{2} \mathrm{O}_{3}$ powder in the ratio 2:3 to insulate the steel/tungsten gaskets, and Daphne oil as the pressure medium. An apparent metal-insulator transition was originally reported at the $90 \mathrm{~K}$ Pnma to Imma transition from resistivity measurements on polycrystalline $\mathrm{PbRuO}_{3}$ samples. ${ }^{19}$ This feature is reproducible but our subsequent studies have shown that it is a microstructural artifact caused by breaking of intergrain or grain-electrode contacts at the first-order structural transition. Application of a few kilobars of pressure suppresses this effect, and the Imma phase is observed to be metallic as reported in other studies. ${ }^{20,21}$ This is also consistent with the small difference in minority spin electron populations of the $t_{2 \mathrm{~g}}$ orbital set

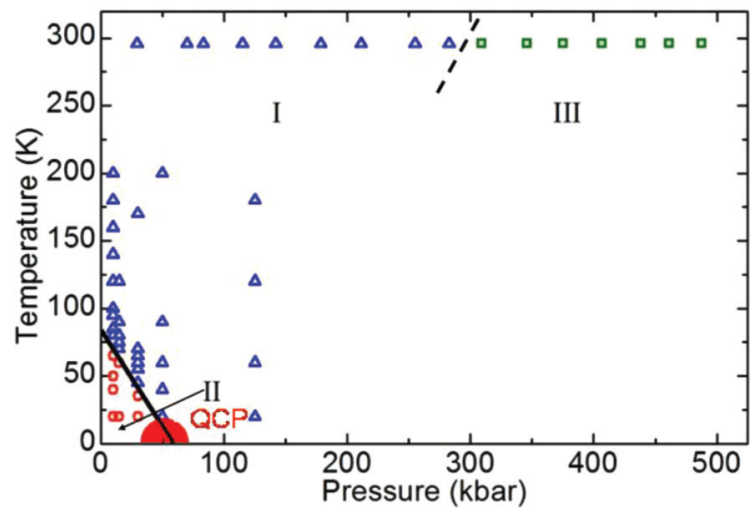

FIG. 2. (Color online) High-pressure phase diagram for $\mathrm{PbRuO}_{3}$ showing the ambient metallic Pnma phase I, the low-temperature orbitally ordered metallic Imma phase II, and the poorly metallic or semiconducting phase III discovered at high pressures. The I-III transition has only been measured at $300 \mathrm{~K}$ so a nominal boundary is shown as a broken line. The critical region around the proposed $50 \mathrm{kbar}$ QCP is also shown. (a)
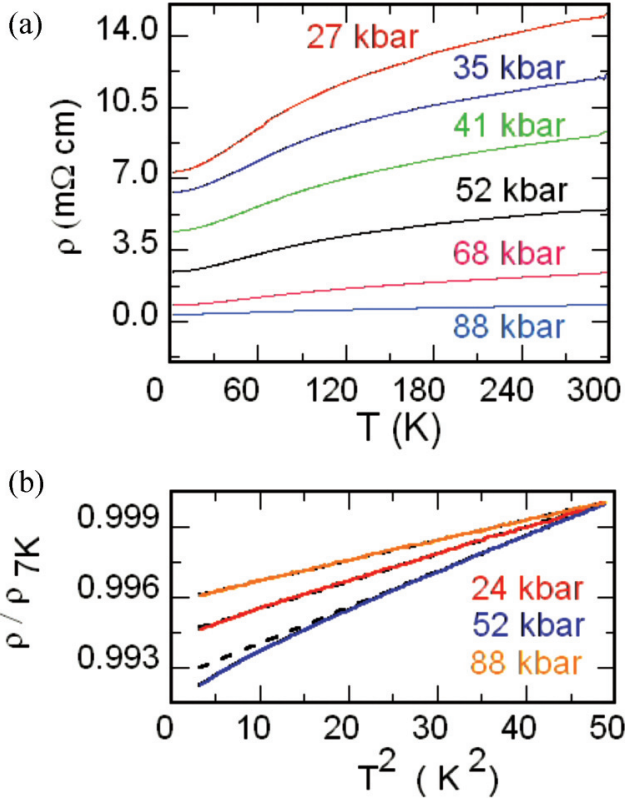

FIG. 3. (Color online) (a) Resistivity of $\mathrm{PbRuO}_{3}$ as a function of temperature at representative pressures between 20 and $90 \mathrm{kbar}$. (b) Plots of $1.8-7 \mathrm{~K}$ resistivities relative to $7 \mathrm{~K}$ values against $T^{2}$, showing good Fermi liquid behavior at 24 and 88 kbar, but a significant deviation at $52 \mathrm{kbar}$ close to the identified QCP.

calculated for the Imma structure. ${ }^{19}$ Hence the I-II transition in $\mathrm{PbRuO}_{3}$ is identified as an orbital ordering in a metallic oxide (a band Jahn-Teller distortion) without an associated spin order or charge localization. The order parameter is the difference in minority spin populations between the $d_{x y}$ and $d_{x z}, d_{y z}$ orbitals. Orbitally ordered metallic states are reported in other perovskite oxides, for example, $\mathrm{PrBaMn}_{2} \mathrm{O}_{6}$ and $\mathrm{NdBaMn}_{2} \mathrm{O}_{6}{ }^{24}$

Resistivities of a $\mathrm{PbRuO}_{3}$ crystal between 20 and 90 kbar pressure are shown in Fig. 3. Smooth temperature variations are obtained without a discontinuity at the I-II orbital ordering transition. Resistivity decreases with increasing pressure in this range, and the residual values of $<1 \mathrm{~m} \Omega \mathrm{cm}$ above $\sim 60$ kbar approach those of a good metal. A change in the low-temperature resistivity variation evidences quantum critical behavior around the $\sim 50 \mathrm{kbar}$ suppression of orbital ordering. All of the results we show in Figs. 3 and 4 are from one DAC experiment, to ensure comparability of data, but measurements on other crystals show the same $50 \mathrm{kbar}$ discontinuity. At pressures well above or below $50 \mathrm{kbar}$ [shown for 24 and 88 kbar data in Fig. 3(b)], resistivity $\rho$ has a quadratic $\rho \sim T^{2}$ variation with temperature $T$, as expected for a conventional Fermi liquid. However, resistivity deviates from $T^{2}$ behavior at an intermediate pressure of $52 \mathrm{kbar}$. To explore this change further, resistivities in the range $1.8-7.0 \mathrm{~K}$ from 12 separate measurements at 30-90 kbar were fitted as $\rho=\rho_{0}+A T^{n}$. Values of the residual resistivity $\rho_{0}$ and thermal exponent $n$ shown in Fig. 4 were obtained from these fits in which $\rho_{0}, n$, and $A$ were varied. A clear discontinuity in $n$ is observed in Fig. 4 , as the exponent falls from $n \approx 1.8$ at pressures near $30 \mathrm{kbar}$ to a minimum value of $n=1.6$ at $50 \mathrm{kbar}$ which evidences non-Fermi liquid behavior. Conventional Fermi liquid behavior is recovered as the pressure is increased 


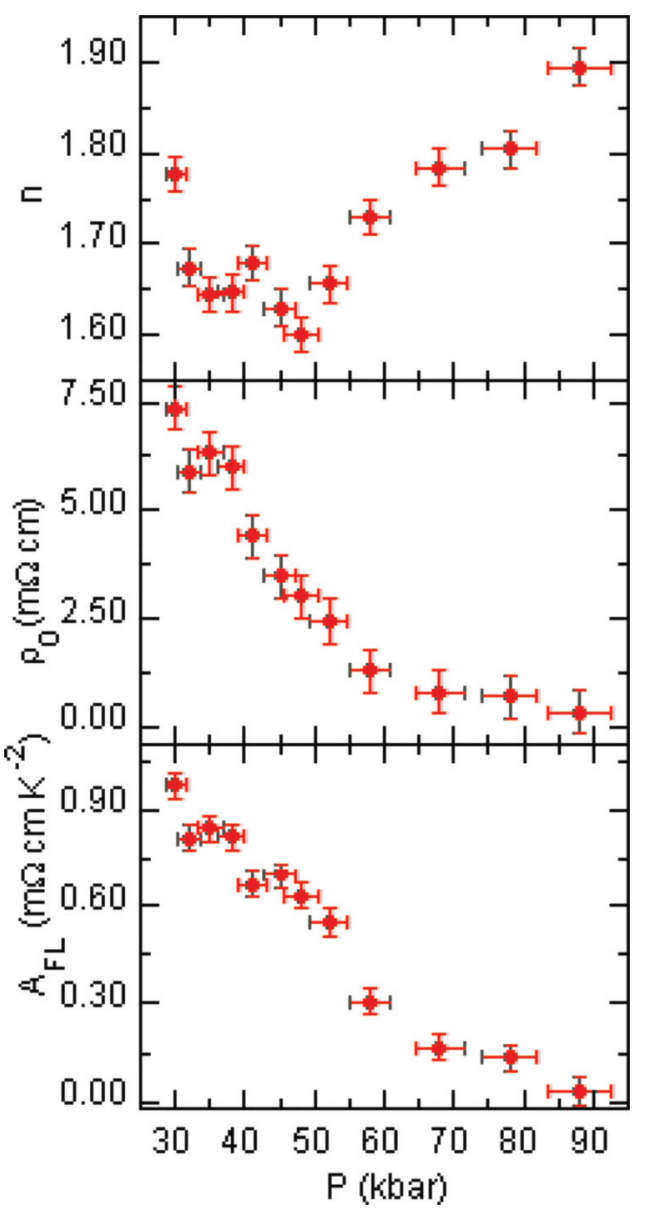

FIG. 4. (Color online) Pressure evolution of parameters extracted from fitting $1.8-7 \mathrm{~K}$ resistivity data as $\rho=\rho_{0}+A T^{n}$ : (a) thermal exponent $n$; (b) residual resistivity $\rho_{0}$; and (c) coefficient $A=A_{\mathrm{FL}}$ obtained when $n$ is fixed to the Fermi liquid $n=2$ value at the lowest temperatures. Anomalies in all these parameters evidence an order parameter change and possible QCP at a critical pressure of $p_{\mathrm{c}} \approx 50 \mathrm{kbar}$.

above $p_{\mathrm{c}}$ and the resistivity exponent approaches the Fermi liquid value of $n=2$. The minimum in $n$ is observed at $48 \mathrm{kbar}$, close to the expected suppression of the I-II structural and orbital ordering transition, and thus evidences a possible QCP at a critical pressure $p_{\mathrm{c}} \approx 50 \mathrm{kbar}$.

A separate series of fits in which the thermal exponent was fixed at $n=2$ were used to extract values of the coefficient $A_{\mathrm{FL}}$ in the Fermi liquid limit from the resistivity data for $\mathrm{PbRuO}_{3}$. The fitting range was significantly reduced around the critical pressure $p_{\mathrm{c}} \approx 50$ kbar to allow convergence of $A_{\mathrm{FL}}$. Peaklike anomalies in the coefficient $A_{\mathrm{FL}}$ and the residual resistivity $\rho_{0}$ are predicted at QCPs associated with suppressed magnetic transitions in metallic materials. ${ }^{25}$ These result from a significant increase of conduction electron (quasiparticle) mass $m^{*}$ near a QCP due to slow, long-range magnetic fluctuations. Peaklike anomalies in $A_{\mathrm{FL}}$ and $\rho_{0}$ were seen from analysis of single crystal resistivities for metamagnetic $\mathrm{Sr}_{3} \mathrm{Ru}_{2} \mathrm{O}_{7}{ }^{6}$ and the heavy fermion ferromagnet $\mathrm{UGe}_{2}$ (Ref. 3) at their QCPs. However, different behaviors are apparent in our $\mathrm{PbRuO}_{3}$ data (Fig. 4), as an anomalous decrease in $A_{\mathrm{FL}}$ and a change of slope of $\rho_{0}$ with pressure are observed
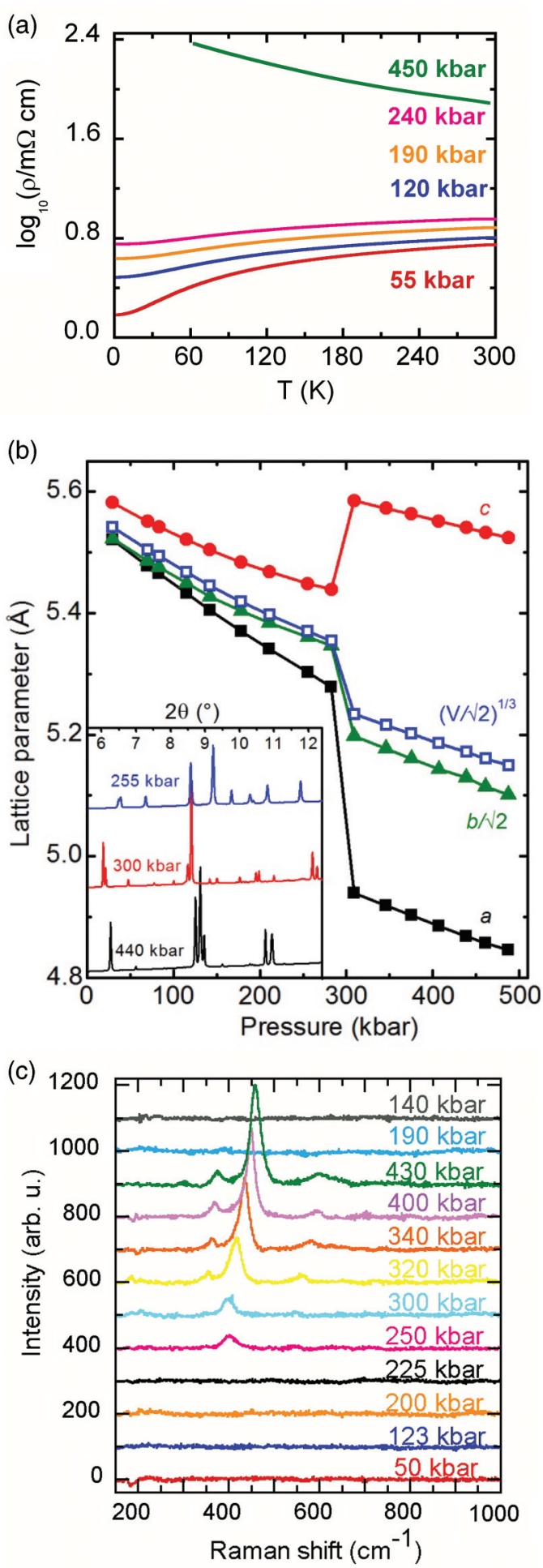

FIG. 5. (Color online) High-pressure measurements across the I-III phase boundary for $\mathrm{PbRuO}_{3}$ at $300 \mathrm{~K}$. (a) $\log$ (resistivity) data showing an increase of resistivity within the metallic phase I up to $240 \mathrm{kbar}$, and apparent semiconducting behavior at $450 \mathrm{kbar}$ for phase III. (b) Orthorhombic cell parameters showing a discontinuity and volume reduction at the transition, with representative $\mathrm{x}$-ray diffraction patterns shown in the inset. (c) Raman spectra on compression from 50 to $430 \mathrm{kbar}$ and during decompression (top two spectra). The appearance of peaks at pressures $>250$ kbar corroborates the poorly metallic or semiconducting nature of phase III. 
at $\sim 55$ kbar. This may signify the emergence of some different scattering mechanism in the vicinity of an orbital QCP, or indicate that a more simple change in an order parameter is occurring. However, the underlying changes may not be observed as impurity scattering can mask possible QCP anomalies, as high-pressure growth does not yield high-purity $\mathrm{PbRuO}_{3}$ crystals [residual resistance ratios $R(300 \mathrm{~K}) / R(2 \mathrm{~K})$ are $<10$ in our crystals, whereas ratios $>100$ were reported for floating-zone crystals of $\mathrm{Sr}_{3} \mathrm{Ru}_{2} \mathrm{O}_{7}$ (Ref. 6)]. Although the intrinsic $A_{\mathrm{FL}}$ and $\rho_{0}$ behaviors are unclear, the pressure variation of $n$ clearly suggests that suppression of orbital order, without an associated magnetic order, leads to a QCP-like feature in metallic $\mathrm{PbRuO}_{3}$. The observed minimum value of $n=1.6$ is close to the $n=5 / 3$ prediction for three-dimensional ferromagnets ${ }^{26}$ - this may be applicable to $\mathrm{PbRuO}_{3}$ as the Imma structure is the orbital analog of a ferromagnet, with a ferro-orbital order of excess $t_{2 \mathrm{~g}}$ electron density in $d_{x y}$ orbitals at all $\mathrm{Ru}$ sites. ${ }^{19}$ Whether the first-order orbital order transition becomes second order between $30 \mathrm{kbar}$ and $p_{\mathrm{c}}$, or remains first order as observed in some correlated electron ferromagnets, ${ }^{3,4}$ is not clear and will require further low-temperature structural measurements close to the quantum critical region.

To explore the possible emergence of a new electronic order above $p_{\mathrm{c}}$, further DAC resistivity measurements were made at pressures $>100$ kbar [Fig. 5(a)]. Surprisingly, these revealed that $\mathrm{PbRuO}_{3}$ becomes more resistive between 120 and 240 kbar. Measurements at higher pressures are challenging, but a successful experiment using small culet diamonds at $450 \mathrm{kbar}$ found a negative $\rho-T$ slope in the measured $70-290 \mathrm{~K}$ range, evidencing semiconducting behavior with an energy gap of $\sim 10 \mathrm{meV}$. The electron-electron correlations responsible for the rise in resistivity beyond 100 kbar do not immediately drive a transition to a new long-range order, as no unexpected distortion of the Pnma phase was observed between 20 and $300 \mathrm{~K}$ at $120 \mathrm{kbar}$ (Fig. 2). However, further DAC synchrotron diffraction data recorded at $300 \mathrm{~K}$ and pressures up to $480 \mathrm{kbar}$ reveal a further structural phase transition at $300 \mathrm{kbar}$ [Fig. 5(b)]. The lattice parameters of this high-pressure phase III are still those of a $\sqrt{2} \times 2 \times \sqrt{2}$ perovskite superstructure, but with a far greater dispersion of magnitudes than in phases I or II showing that the perovskite arrangement is highly distorted. A substantial (11\%) volume reduction is observed at the first-order I-III structural transition. The X-ray diffraction peaks from the $>300$ kbar phase III have reflection conditions consistent with primitive orthorhombic space group Pnna. However, it was not possible to refine a structural model because of the granularity or texturing effects noted above, and further studies will be needed to determine the full structure of phase III.

The I-III transition in $\mathrm{PbRuO}_{3}$ is confirmed by Raman spectroscopy [Fig. 5(c)]. Spectra from polycrystalline $\mathrm{PbRuO}_{3}$ in a Merrill-Bassett-type DAC cell were recorded at $300 \mathrm{~K}$ with a 4:1 mixture of ethanol and methanol as the pressure medium and a ruby as a pressure calibrant. The spectrum of the ambient Pnma phase I is featureless in the 100$1000 \mathrm{~cm}^{-1}$ frequency range, but sharp peaks emerge at the 250-300 kbar approach to the I-III transition and persist to the highest measured pressure of $430 \mathrm{kbar}$. This corroborates the change from metallic to a more resistive behavior found in transport measurements [Fig. 5(a)]. The changes observed in the Raman spectra are reversible, as shown at the top of Fig. 5(c), confirming that they have not resulted from sample amorphization or decomposition.

The increased resistivity upon pressurization and possible opening of a gap at $300 \mathrm{kbar}$ in $\mathrm{PbRuO}_{3}$ is very unusual as displacive transitions driven by pressure usually result in more highly conducting phases. The resistivity measurements in Fig. 5(a) show that resistive correlations are evident above pressures of at least $120 \mathrm{kbar}$, and so may emerge from the vicinity of the implied $50 \mathrm{kbar}$ QCP. Full structure determination of the high-pressure phase III is needed to identify the emergent order. A (non-ferro-) orbital order, perhaps coupled to $\mathrm{Ru}^{4+}$ spin order, or an array of $\mathrm{Ru}-\mathrm{O}-\mathrm{Ru}$ spin singlet dimers like those in $\mathrm{La}_{4} \mathrm{Ru}_{2} \mathrm{O}_{10}$ (Ref. 27) are possible ground states.

\section{CONCLUSIONS}

This study demonstrates that $\mathrm{PbRuO}_{3}$ may exemplify a long-range orbital ordering transition driven to a QCP in an itinerant electron material. The observed minimum value of the temperature exponent as the ferro-orbital order is suppressed is close to the $n=5 / 3$ prediction for three-dimensional ferromagnets. The possibility for new orbital physics is demonstrated by an anomalous increase in resistivity at pressures beyond $p_{\mathrm{c}}$, and the emergence of a further superstructure phase III that may be a poor metal or a semiconductor. The origin of the proposed QCP in $\mathrm{PbRuO}_{3}$ is different from those in $\mathrm{Sr}_{3} \mathrm{Ru}_{2} \mathrm{O}_{7}$ and heavy fermion metals, which are usually accessed by driving a magnetic transition towards zero temperature. However, the presence of strong-spin orbit coupling in such materials suggests that spin and orbital quantum criticality are ultimately connected, as illustrated by the recently reported emergence of nematic orbital order around the QCP in $\mathrm{Sr}_{3} \mathrm{Ru}_{2} \mathrm{O}_{7} .{ }^{8}$ Hence, magnetism may be involved around the QCP or in the high-pressure phase III of $\mathrm{PbRuO}_{3}$. Further experimental studies of $\mathrm{PbRuO}_{3}$ may help to guide theories of orbital criticality ${ }^{28}$ and their application to other orbitally ordered materials such as iron pnictide superconductors, ${ }^{29}$ but they present challenges to growing cleaner crystals and measure resistivity and magnetization accurately at high pressures.

\section{ACKNOWLEDGMENTS}

We thank Michael Hanfland for assistance with ESRF data collection; EPSRC, the Leverhulme Trust, and the Royal Society for financial support; and STFC for access to ESRF.
*Corresponding author: j.p.attfield@ed.ac.uk
${ }^{1}$ Q. Si and F. Steglich, Science 329, 1161 (2010).
${ }^{2}$ T. Takami, Jpn. J. Appl. Phys. 51, 010004 (2012).
${ }^{3}$ S. S. Saxena, P. Agarwal, K. Ahilan, F. M. Grosche, R. K. W. Haselwimmer, M. J. Steiner, E. Pugh, I. R. Walker, S. R. Julian, P. Monthoux, G. G. Lonzarich, A. Huxley, 
I. Sheikin, D. Braithwaite, and J. Flouquet, Nature 406, 587 (2000).

${ }^{4}$ E. A. Yelland, J. M. Barraclough, W. Wang, K. V. Kamenev, and A. D. Huxley, Nat. Phys. 7, 890 (2011).

${ }^{5}$ S. Maekawa, T. Tohyama, S. E. Barnes, S. Ishihara, W. Koshibae, and G. Khaliullin, Physics of Transition Metal Oxides (Springer, Berlin, 2004).

${ }^{6}$ S. A. Grigera, R. S. Perry, A. J. Schofield, M. Chiao, S. R. Julian, G. G. Lonzarich, S. I. Ikeda, Y. Maeno, A. J. Millis, and A. P. Mackenzie, Science 294, 329 (2001).

${ }^{7}$ S. A. Grigera, P. Gegenwart, R. A. Borzi, F. Weickert, A. J. Schofield, R. S. Perry, T. Tayama, T. Sakakibara, Y. Maeno, A. G. Green, and A. P. Mackenzie, Science 306, 1154 (2004).

${ }^{8}$ C. Stingl, R. S. Perry, Y. Maeno, and P. Gegenwart, Phys. Rev. Lett. 107, 026404 (2011).

${ }^{9}$ M. K. Jacobsen, R. S. Kumar, G. Cao, J. J. Neumeier, and A. L. Cornelius, J. Phys. Chem. Solids 69, 2237 (2008).

${ }^{10}$ K. Ishida, H. Mukuda, Y. Kitaoka, K. Asayama, Z. Q. Mao, Y. Mori, and Y. Maeno, Nature 396, 658 (1998).

${ }^{11}$ S. Nakatsuji, S.-I. Ikeda, and Y. Maeno, J. Phys. Soc. Jpn. 66, 1868 (1997).

${ }^{12}$ F. Nakamura, J. Phys. Soc. Jpn. 76, 96 (2007).

${ }^{13}$ P. L. Alireza, F. Nakamura, S. K. Goh, Y. Maeno, S. Nakatsuji, Y. T. C. Ko, M. Sutherland, S. R. Julian, and G. G. Lonzarich, J. Phys.: Condens. Matter 22, 052202 (2010).

${ }^{14}$ J. J. Randall and R. Ward, J. Am. Chem. Soc. 81, 2629 (1959).

${ }^{15}$ J. B. Goodenough, Czech. J. Phys. B 17, 304 (1967).

${ }^{16}$ C.-Q. Jin, J.-S. Zhou, J. B. Goodenough, Q. Q. Liu, J. G. Zhao, L. X. Yang, Y. Yu, R. C. Yu, T. Katsura, A. Shatskiy, and E. Ito, Proc. Natl. Acad. Sci. U.S.A. 105, 7115 (2008).

${ }^{17}$ G. Cao, S. McCall, M. Shepard, J. E. Crow, and R. P. Guertin, Phys. Rev. B 56, 321 (1997).

${ }^{18}$ N. Kikugawa, L. Balica, and A. P. Mackenzie, J. Phys. Soc. Jpn. 78, 014701 (2009).

${ }^{19}$ S. A. J. Kimber, J. A. Rodgers, H. Wu, C. A. Murray, D. N. Argyriou, A. N. Fitch, D. I. Khomskii, and J. P. Attfield, Phys. Rev. Lett. 102, 046409 (2009).

${ }^{20}$ J.-G. Cheng, J.-S. Zhou, and J. B. Goodenough, Phys. Rev. B 80, 174426 (2009).

${ }^{21}$ J.-G. Cheng, J.-S. Zhou, and J. B. Goodenough, Phys. Rev. B 81, 134412 (2010).

${ }^{22}$ J. A. Kafalas and J. M. Longo, Mater. Res. Bull. 5, 193 (1970).

${ }^{23}$ A. C. Larson and R. B. Von Dreele, "General Structure Analysis System (GSAS)", Los Alamos National Laboratory Report LAUR 86-748 (1994).

${ }^{24}$ T. Nakajima, H. Kageyama, H. Yoshizawa, K. Ohoyama, and Y. Ueda, J. Phys. Soc. Jpn. 72, 3237 (2003).

${ }^{25}$ G. G. Lonzarich and L. Taillefer, J. Phys. C: Solid State Phys. 18, 4339 (1985).

${ }^{26}$ T. Moriya and K. Ueda, Adv. Phys. 49, 555 (2000).

${ }^{27}$ H. Wu, Z. Hu, T. Burnus, J. D. Denlinger, P. G. Khalifah, D. G. Mandrus, L.-Y. Jang, H. H. Hsieh, A. Tanaka, K. S. Liang, J. W. Allen, R. J. Cava, D. I. Khomskii, and L. H. Tjeng, Phys. Rev. Lett. 96, 256402 (2006).

${ }^{28}$ K. W. Lo, W.-C. Lee, and P. W. Phillips, arXiv:1207.4206 (2012).

${ }^{29}$ C.-C. Chen, J. Maciejko, A. P. Sorini, B. Moritz, R. R. P. Singh, and T. P. Devereaux, Phys. Rev. B 82, 100504(R) (2010). 